\title{
CHARACTERIZATION OF THE TEMPORAL AND SPATIAL DYNAMICS OF THE DENGUE EPIDEMIC IN NORTHERN SRI LANKA
}

\author{
S. Anno ${ }^{\text {a, }}{ }^{*}$, K. Imaoka ${ }^{\text {b }}$, T. Tadono ${ }^{\text {b }}$, T. Igarashi ${ }^{\text {c }}$, S. Sivaganesh ${ }^{\text {d }}$, S. Kannathasan ${ }^{\text {e }}$, V. Kumaran ${ }^{\text {e }}$, S.N. Surendran ${ }^{\text {e }}$ \\ ${ }^{\text {a }}$ Shibaura Institute of Technology, Tokyo, Japan - annou@ @ic.shibaura-it.ac.jp \\ b Japan Aerospace Exploration Agency, Ibaraki, Japan - imaoka.keiji@jaxa.jp; tadono.takeo@jaxa.jp \\ ${ }^{\mathrm{c}}$ Remote Sensing Technology Center of Japan, Tokyo, Japan - igarashi_tamotsu@ restec.or.jp \\ ${ }^{\mathrm{d}}$ Regional Epidemiologist, Jaffna, Sri Lanka - sgh1210@yahoo.com \\ e University of Jaffna, Jaffna, Sri Lanka - selvamkannathasan@gmail.com; vaithehik25@yahoo.com; noble@jfn.ac.lk
}

\section{Commission VI, WG VI/4}

KEY WORDS: Dengue, Ecological and socio-economic and demographic factors, Local Moran LISA statistics, Temporal analysis, Spatial association analysis, Spatial statistical analysis

\begin{abstract}
:
Dengue outbreaks are affected by biological, ecological, socio-economic and demographic factors that vary over time and space. These factors have been examined separately, with limited success, and still require clarification. The present study aimed to investigate the spatial and temporal relationships between these factors and dengue outbreaks in the northern region of Sri Lanka. Remote sensing (RS) data gathered from a plurality of satellites: TRMM TMI, Aqua AMSR-E, GCOM-W AMSR2, DMSP SSM/I, DMSP SSMIS, NOAA-19 AMSU, MetOp-A AMSU and GEO IR were used to develop an index comprising rainfall. Humidity (total precipitable water, or vertically integrated water vapor amount) and temperature (surface temperature) data were acquired from the JAXA Satellite Monitoring for Environmental Studies (JASMES) portal which were retrieved and processed from the Aqua/MODIS and Terra/MODIS data. RS data gathered by ALOS/AVNIR-2 were used to detect urbanization, and a digital land cover map was used to extract land cover information. Other data on relevant factors and dengue outbreaks were collected through institutions and extant databases. The analyzed RS data and databases were integrated into geographic information systems, enabling both spatial association analysis and spatial statistical analysis. Our findings show that the combination of ecological factors derived from RS data and socio-economic and demographic factors is suitable for predicting spatial and temporal patterns of dengue outbreaks.
\end{abstract}

\section{INTRODUCTION}

Dengue is the most important vector-borne viral disease worldwide and a major cause of childhood fever burden in Sri Lanka, which has experienced a number of large epidemics in the past decade (Tam et al., 2013). Dengue is now considered to be hyperendemic in Sri Lanka, with detected co-circulation of multiple serotypes (Kanakaratne, 2009; WHO, 2011).

Dengue outbreaks are affected by biological, ecological, socioeconomic and demographic factors that vary over time and space. Disease-promoting factors include 1) climate, such as rainfall, humidity and temperature (Canyon, 1999); 2) changes in land cover, particularly rapid unplanned expansion of urbanization with inadequate housing and infrastructure (Gubler, 1997; Rodhain and Rosen, 1997; Lian, et al., 2006; Ooi and Gubler, 2008; Tran, et al. 2010; Gubler, 2011; WHO, 2012); and 3) high population density (Gubler, 1998).

Previous studies have identified a large number of biological, ecological and socio-economic and demographic factors that are considered to impact both susceptibility and exposure to spatial and temporal outbreaks of dengue in Sri Lanka. However, these factors have mainly been examined separately, with limited success. Further clarification is required. The present study aimed to investigate the spatial and temporal relationships between these factors and outbreaks of dengue associated with mosquito breeding sites and habitats in northern Sri Lanka.

\section{MATERIAL AND METHODS}

\subsection{Study Area}

Our study area was the northern region of Sri Lanka, consisting of twelve Medical Officer of Health (MOH) divisions which are the health administrative divisions in Sri Lanka. Each $\mathrm{MOH}$ division has different geographic features-including agricultural fields, forested areas, wetlands, grassland, urban areas, etc. - as well as different social backgrounds. The climate in the region is tropical, with two monsoon seasons: namely north east monsoon from November to April, and south west monsoon from May to October.

\subsection{Dengue Data}

From the MOH divisions in Sri Lanka, we obtained the monthly numbers of clinically confirmed dengue cases from January 2010 through December 2013 in the twelve MOH divisions, and the annual numbers of clinically confirmed dengue cases from 2007 through 2013 in the same MOH divisions.

\subsection{Rainfall, Humidity and Temperature}

Rainfall data were obtained using the Global Satellite Mapping of Precipitation (GSMaP) product, based on the combined MWIR algorithm with a plurality of satellites: TRMM TMI, Aqua AMSR-E, GCOM-W AMSR2, DMSP SSM/I, DMSP SSMIS, NOAA-19 AMSU, MetOp-A AMSU and GEO IR. The GSMap 
is drawn to the highest levels of precision and resolution in the world (Temporal resolution: 1 hour, Spatial resolution: Grid latitude-longitude of 0.1 degrees) (JAXA, 2014a; RESTEC, 2014). The near-real-time version (GSMaP_NRT) is published with an approximately 4-hour time-lag from the time of satellite observation, and the reanalysis version (GSMaP_MVK) is available after the reprocessing is completed. The monthly average rainfall from January 2010 to December 2013 and the annual average rainfall from 2007 to 2013 were obtained by processing the GSMaP_MVK and GSMaP_NRT data depending on the period, and converting this processed data into TIFF image data for spatial analysis in geographic information systems (GIS).

Humidity (total precipitable water, or vertically integrated water vapor amount) and temperature (surface temperature) data were acquired from the JAXA Satellite Monitoring for Environmental Studies (JASMES) portal (JAXA, 2014b). These geophysical parameters were retrieved and processed from the Aqua/MODIS and Terra/MODIS data. The MODIS instrument is operating on both the Terra and Aqua spacecraft. It has a viewing swath width of 2,330 km, and views the entire Earth's surface every one to two days. Its detectors measure 36 spectral bands between 0.405 and $14.385 \mu \mathrm{m}$, and it acquires data at three spatial resolutions: $250 \mathrm{~m}, 500 \mathrm{~m}$ and 1,000 m (NASA, 2014). We obtained the monthly averages from January 2010 to December 2013, and the annual averages from 2007 to 2013 by processing the RS data, and converting these processed data into TIFF image data for spatial analysis in GIS.

\subsection{Land Cover, Including Urbanization}

A paper survey map was digitized to generate detailed land cover data, and the digital land cover map of this study area was used for spatial analysis in GIS.

The ALOS/AVNIR-2 data set was used to detect urbanization by conducting unmixing, which isolates the contribution of a specific material within the mixed pixel. This method identifies the locations of pixels that contain a given material, and reports the material pixel fraction, i.e., how much of the material is in each pixel. We selected eight material pixel fraction classes that report subpixel detections in material pixel fraction increments of 0.20. Pixels determined to have material pixel fractions of 20-29\% belong to class $0.20-0.29$ and pixels with material pixel fractions of $90-100 \%$ belong to class $0.90-1.00$.

\subsection{Population Density}

We obtained annual population data for the respective $\mathrm{MOH}$ division from 2007 to 2013 from the Regional Epidemiological Unit, Jaffna. With this information combined with the area data by $\mathrm{MOH}$ division obtained from spatial analysis in GIS, we calculated the population density as a socio-economic and demographic factor. We also calculated the average value from a set of annual population density at the $\mathrm{MOH}$ division level to investigate the comprehensive trend of annual population density. This was used for both spatial association analysis and spatial statistical analysis.

\subsection{Incidence Rates}

To examine the spatial patterns of dengue disease, epidemic curves were produced by calculating the annual dengue incidence rate during the period 2007-2013. Annual incidence rates for each $\mathrm{MOH}$ division were calculated from the number of annual confirmed dengue cases, divided by the total population-years and then multiplied by 10,000 . These rates were expressed as annual confirmed dengue cases divided by total population*10,000 people. The average annual incidence at the MOH division level was also calculated to determine the comprehensive trend of annual incidence. This was used for both spatial association analysis and spatial statistical analysis.

\subsection{Spatial Analysis in GIS}

A polygon layer that generates the twelve $\mathrm{MOH}$ divisions in the northern region of Sri Lanka was used for spatial analysis in GIS. TIFF image data on rainfall, humidity and temperature were integrated into GIS, and the pixel (i.e., raster) size was changed from 0.05 to 0.01 . The polygon layer and the processed raster layer were overlaid. The values of a raster within the polygons were summarized, and the results were reported to excel tables.

The digital land cover map was integrated into GIS. The polygon layer and the digital land cover map layer were overlaid. The land cover data within the polygons were summarized and the results were reported to excel tables. The raster data on urbanization were integrated into GIS. The polygon layer and the raster layer were overlaid. Again, the values of a raster within the polygons were summarized, and the results were reported to excel tables.

The table of polygon layer attributes was joined with the excel tables containing data on ecological, socio-economic and demographic factors. This information was used for spatial association analysis and spatial statistical analysis.

We additionally calculated the average values from data on annual rainfall, humidity and temperature at $\mathrm{MOH}$ division level. This information was used to investigate the comprehensive trend of annual rainfall, humidity and temperature, and was used for both spatial association analysis and spatial statistical analysis.

\subsection{Temporal Analysis}

To examine temporal patterns, we used data on monthly dengue cases, rainfall, humidity and temperature during the period from January 2010 through December 2013. The moving average (MA) was calculated and visualized to examine the temporal climate trend associated with outbreak of dengue. We also calculated the average monthly values from data on monthly rainfall, humidity and temperature within the period to investigate the comprehensive trend and to be used for the chisquare test. The chi-square test was used to test monthly differences in dengue cases, rainfall, humidity and temperature across the study period. The statistical significance was set at 0.05 .

\subsection{Spatial Association Analysis}

Univariate Local Indicators of Spatial Association (LISA) was applied to measure the local spatial autocorrelation of dengue outbreak using GeoDa (Anselin, 1995; Anselin et al., 2006). LISA are statistics that measure spatial dependence and evaluate the existence of local clusters within the spatial arrangement of a given variable. They are based on a statistical index I developed by Moran to measure the global spatial autocorrelation of the overall data clustering in the area under investigation (Moran, 1950). Moran's I ranges from -1 
(negative spatial autocorrelation) to 1 (complete spatial dependence), with 0 indicating an absence of spatial dependence (i.e., random distribution) (Guessous et al., 2014). Local spatial autocorrelation analysis was performed based on the Local Moran LISA statistics, which yields a measure of spatial autocorrelation for each individual location. The LISA statistic reveals five categories of spatial autocorrelation that appear on the cluster map legend: 1) not significant (i.e., areas that are not significant at the default pseudo-significance level of 0.05$), 2$ ) high-high (high values surrounded by high values), 3) low-low (low values surrounded by low values), 4) low-high (low values surrounded by high values) and 5) high-low (high values surrounded by low values) (Anselin, 2003; Singh et al., 2011). High-high and low-low represent positive spatial autocorrelation (i.e., association between areas of similar values), and high-low and low-high represent negative spatial correlation (i.e., association between areas of dissimilar values) (Anselin, 2003; Martinez et al., 2014). A finding of significant clustering at $\mathrm{p}<0.05$ suggests that dengue incidence values are too similar across these neighboring provinces to have occurred by chance, providing significant evidence for rejecting the null hypothesis of spatial randomness (Martinez et al., 2014).

\subsection{Spatial Statistical Analysis}

The chi-square test was used to test the spatial association between ecological, socio-economic and demographic factors and dengue outbreak. The statistical significance was set at 0.05 . As a second exploratory analysis, using the results from the chisquare test, we compared the differences in ecological, socioeconomic and demographic factors between the areas of significant high-high clustering (i.e., endemic areas) and the areas of significant low-high clustering (i.e., non-endemic areas estimated to be controlled by some factors) as identified in the univariate LISA analysis.

\section{RESULTS}

\subsection{Results of Temporal Analysis}

Humidity tends to rise in early January, remaining high during the dry season, and then declining with the increase in rainfall in early September. These changes are accelerated at lower temperature. The distribution of monthly dengue cases indicated a strong seasonal pattern. Dengue case tended to increase after exponential increases or decreases in rainfall. The chi-square test results supported these tendencies. We observed significant monthly differences in dengue cases and rainfall $(\mathrm{p}<0.01)$, while humidity and temperature were not significant.

\subsection{Results of Spatial Association Analysis}

The LISA cluster map shows two types of geographical clustering (high-high and low-high). The area of significant high-high clustering of the average values from a set of annual dengue incidences accounted for $8.3 \%$ and occurred in Nallur $\mathrm{MOH}$ division. The area of significant low-high clustering of the average values from a set of annual dengue incidences accounted for $16.7 \%$ and occurred in Kopay and Tellipallai $\mathrm{MOH}$ divisions. The Moran's I statistic was -0.08 , suggesting a random spatial pattern.

\subsection{Results of Spatial Statistical Analysis}

The spatial statistical analysis revealed the dengue outbreak was significantly associated with ecological, socio-economic and demographic factors. Significantly more dengue cases were observed in $\mathrm{MOH}$ divisions $(66.7 \%)$ with average annual rainfall of $>1353 \mathrm{~mm}$ compared to in those with average annual rainfall of $<1353 \mathrm{~mm}\left(\chi^{2}=112.8 ; \mathrm{p}<0.01\right)$. Correspondingly, we also observed significantly more dengue cases in $\mathrm{MOH}$ divisions (66.7\%) with average annual humidity of $>39.62 \mathrm{~mm}$ compared to in those with average annual humidity of $<39.62$ $\mathrm{mm}\left(\chi^{2}=55.6 ; \mathrm{p}<0.01\right)$. Moreover, significantly more dengue cases occurred in $\mathrm{MOH}$ divisions $(58.3 \%)$ with an average annual temperature of $>31.2^{\circ} \mathrm{C}$ compared to in those with an average annual temperature of $<31.2^{\circ} \mathrm{C}\left(\chi^{2}=104.7\right.$; $\left.<<0.01\right)$.

Dengue occurrence was also significantly associated with the presence or absence of built-up area considered to represent urbanization $\left(\chi^{2}=264.7 ; \mathrm{p}<0.01\right)$. The presence of built-up area in $\mathrm{MOH}$ divisions (33.3\%) significantly influenced dengue occurrence, with significantly more dengue cases observed in MOH divisions $(50.0 \%)$ that had a $>18 \%$ ratio of urbanization to $\mathrm{MOH}$ division area compared to in those with a $<18 \%$ ratio of urbanization to MOH division area $\left(\chi^{2}=40.7\right.$; $\left.<0.01\right)$. We also found significantly more dengue cases in $\mathrm{MOH}$ divisions (33.3\%) with a population density of $>1150$ compared to those with a population density of $<1150\left(\chi^{2}=347.2\right.$; $\left.p<0.01\right)$.

The chi-square test results showed Nallur MOH division to be a high-high clustering area, with built-up land area and a higher population density, while Kopay and Tellipallai $\mathrm{MOH}$ divisions were low-high clustering areas lacking built-up land area and having lower population densities. These results suggest significant differences in the presence or absence of built-up area and higher population density between high-high clustering areas and low-high clustering areas. Presence of built-up area and higher population density could influence the dengue occurrence.

\section{CONCLUSION}

Our results showed that dengue outbreak was associated with rainfall, humidity, temperature, built-up area considered to represent urbanization, urbanization and higher population density. Furthermore, our analyses quantitatively indicated to what degree these factors influenced dengue occurrence. Our findings indicate that these factors impact vulnerability to dengue by creating conditions of either susceptibility within human communities or of exposure to the vector and proximity to breeding habitats.

The spatial and temporal association found in our study highlights the fact that rainfall, humidity, temperature, built-up area, urbanization and higher population density can strengthen forecasting models. Spatial-temporal models must be developed and strengthened by incorporating ecological and socioeconomic and demographic factors for further analysis.

Dengue transmission within Sri Lanka is spatially heterogeneous. Further research must focus on the whole island to improve the accuracy of spatial and temporal models. An integrated spatial-temporal prediction model using ecological and socio-economic and demographic factors could lead to substantial improvements in the control and prevention of dengue by allocating the right resources to the appropriate places at the right time. 


\section{REFERENCES}

Anselin, L., 1995. Local indicators of spatial associationLISA, Geographical Analysis, 27, pp. 93-116.

Anselin, L., 2003. Local Spatial Autocorrelation, In GeoDa ${ }^{\mathrm{TM}}$ 0.9 User's Guide, University of Illinois, Urbana-Champaign, Urbana, IL, pp. 99-101.

Anselin, L, Syabri I and Kho Y., 2006. GeoDa: An introduction to spatial data analysis, Geographical Analysis, 38, pp. 5-22

Canyon, DV, Hii JL and Müller R., 1999. Adaptation of Aedes aegypti (Diptera:Culicidae) oviposition behavior in response to humidity and diet, Journal of Insect Physiology, 45(10), pp. 959-964.

Gubler, DJ., 1997. Dengue and Dengue Hemorrhagic Fever: Its History and Resurgence as a Global Public Health Problem, In: Gubler DJ, and Kuno G, eds., Dengue and Dengue Hemorrhagic Fever, CAB International, London, pp. 1-22.

Gubler, DJ., 1998. Dengue and dengue hemorrhagic fever, Clinical Microbiology Reviews, 11(3), pp. 480-496.

Gubler, DJ., 2011. Dengue, Urbanization and Globalization: The Unholy Trinity of the 21 st Century, Tropical Medicine and Health, 39(4), Supplement, pp. 3-11.

Guessous, I, Joost S, Jeannot E, Theler JM, Mahler P, Gaspoz JM and GIRAPH Group (2014), A comparison of the spatial dependence of body mass index among adults and children in a Swiss general population, Nutrition Diabetes, Vol. 4, pp. e111.

JAXA, 2014a, JAXA Global Rainfall Watch, Available: http://sharaku.eorc.jaxa.jp/GSMaP/index.htm Accessed August 2014

JAXA, 2014b, JAXA Satellite Monitoring for Environmental Studies (JASMES), Available:

http://kuroshio.eorc.jaxa.jp/JASMES/index.html

Accessed August 2014

Kanakaratne, N, Wahala WM, Messer WB, Tissera HA, Shahani A, Abeysinghe N, de-Silva AM and Gunasekera M., 2009. Severe Dengue Epidemics in Sri Lanka, 2003-2006, Emerging Infectious Diseases, 15(2), pp. 192-199.

Lian, CW, Seng CM and Chai WY., 2006. Spatial, environmental and entomological risk factor analysis on a rural dengue outbreak in Lundu District in Sarawak, Malaysia. Tropical Biomedicine, 23, pp. 85-96.

Martinez, AN, Mobley LR, Lorvick J, Novak SP, Lopez AM and Kral AH (2014), Spatial analysis of HIV positive injection drug users in San Francisco, 1987 to 2005, International Journal of Environmental Research and Public Health, 11(4), pp. 3937-3955.

Moran, PAP (1950), Notes on continuous stochastic phenomena, Biometrika, 37, pp. 17-23.

NASA, 2014. National Aeronautics and Space Administration, MODIS Web, Available: http://modis.gsfc.nasa.gov/data/ Accessed August 2014.
Ooi, EE and Gubler DJ., 2008. Dengue in Southeast Asia: epidemiological characteristics and strategic challenges in disease prevention, Cadernos de Saúde Pública, 25, Supplement, pp. 115-124.

RESTEC, 2014. Global Satellite Mapping of Precipitation (GSMaP),

Available: https://www.restec.or.jp/. Accessed August 2014.

Rodhain, F and Rosen L., 1997. Mosquito vectors and dengue virus-vector relationships, In: Gubler DJ, and Kuno G, eds., Dengue and Dengue Hemorrhagic Fever, UK: CAB International, Wallingford, pp. 45-60

Singh, A, Pathak PK, Chauhan RK and Pan W., 2011. Infant and child mortality in India in the last two decades: a geospatial analysis, PLoS One, 6(11), pp. e26856.

Tam, CC, Tissera H, de Silva AM, De Silva AD, Margolis HS and Amarasinge A., 2013. Estimates of Dengue Force of Infection in Children in Colombo, Sri Lanka, PLOS Neglected Tropical Diseases, 7(6), pp. 1-7.

Tran, HP, Adams J, Jeffery JA, Nguyen YT, Vu NS, Kutcher SC, Kay BH and Ryan PA., 2010. Householder perspectives and preferences on water storage and use, with references to dengue, in the Mekong Delta, southern Vietnam, International Health, 2(2), pp. 136-142.

WHO South East Asia Regional Office, 2011. Comprehensive Guidelines for Prevention and Control of Dengue and Dengue Haemorrhagic Fever - Revised and Expanded Edition, New Delhi, Available: http://www.searo.who.int/entity/vector_borne_tropical_diseases /documents/SEAROTPS60/en/index.html Accessed May 2013.

WHO, 2012. Dengue and Severe Dengue, Fact Sheet Nu117, Available: http://www.who.int/mediacentre/factsheets/fs117/en/ Accessed April 2012. 